\title{
VU0606170, a Selective Slack Channels Inhibitor, \\ Decreases Calcium Oscillations in Cultured Cortical Neurons
}

\section{Authors}

Brittany D Spitznagel ${ }^{1}$, Nigam M Mishra ${ }^{2}$, Alshaima'a M Qunies ${ }^{2,3}$, Francis J Prael 3rd ${ }^{1,4}$, Yu Du ${ }^{1,4}$, Krystian A Kozek $^{1,4,5}$, Roman M Lazarenko ${ }^{6}$, Jerod S Denton ${ }^{1,6}$, Kyle A Emmitte ${ }^{2}$, C David Weaver ${ }^{*}, 1,4$

\begin{abstract}
Affiliations
${ }^{1}$ Department of Pharmacology, Vanderbilt University, Nashville, Tennessee 37232, United States.

${ }^{2}$ Department of Pharmaceutical Sciences, UNT System College of Pharmacy, University of North Texas Health Science Center, Fort Worth, Texas 76107, United States.

${ }^{3}$ Graduate School of Biomedical Sciences, University of North Texas Health Science Center, Fort Worth, Texas 76107, United States.

${ }^{4}$ Vanderbilt Institute of Chemical Biology, Vanderbilt University, Nashville, Tennessee 37240, United States. ${ }^{5}$ Vanderbilt Medical Scientist Training Program, Vanderbilt University, Nashville, Tennessee 37232, United States.

${ }^{6}$ Department of Anesthesiology, Vanderbilt University, Nashville, Tennessee 37212, United States.
\end{abstract}

\section{Supplemental Information}

\begin{tabular}{|l|c|}
\hline \multicolumn{1}{|c|}{ CHANNEL } & NUMBER OF HITS \\
\hline Wildtype Slack & 147 \\
\hline G288S Slack & 101 \\
\hline R428Q Slack & 56 \\
\hline A934T Slack & 113 \\
\hline
\end{tabular}

Table S1: Shown are the results of a 4800 compound screening of WT and three Slack mutants in 384-well plates (60 plates). Hits were identified using an automated (WaveGuide) hit picking algorithm (see methods) supplemented with manual spot-checking. When hits from the WT Slack and A934T mutant Slack channels were combined they accounted for $100 \%$ of the hits detected using all four cell lines, therefore the 100,000 compound screen was performed using WT and A934T Slack expressing HEK293 cell lines. 


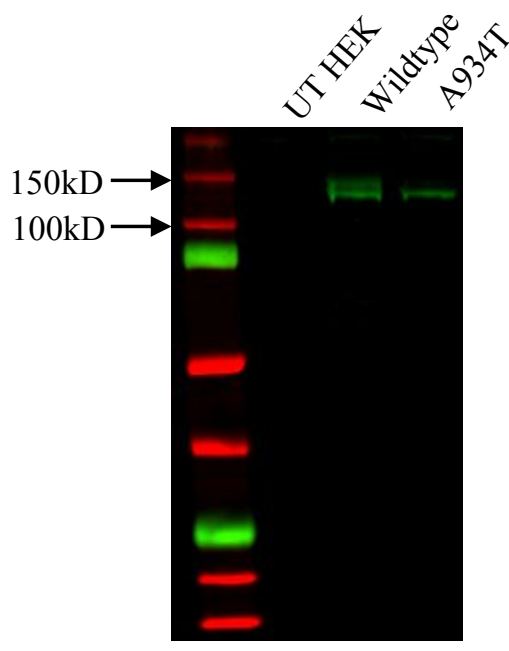

Figure S1: Stable expression of Slack in HEK293 cells. Shown is a Western blot of membrane fractions probed with a Slackspecific antibody: untransfected HEK293 cells, HEK293 cells stably expressing WT Slack, and HEK293 cells stably expressing A934T mutant Slack. 


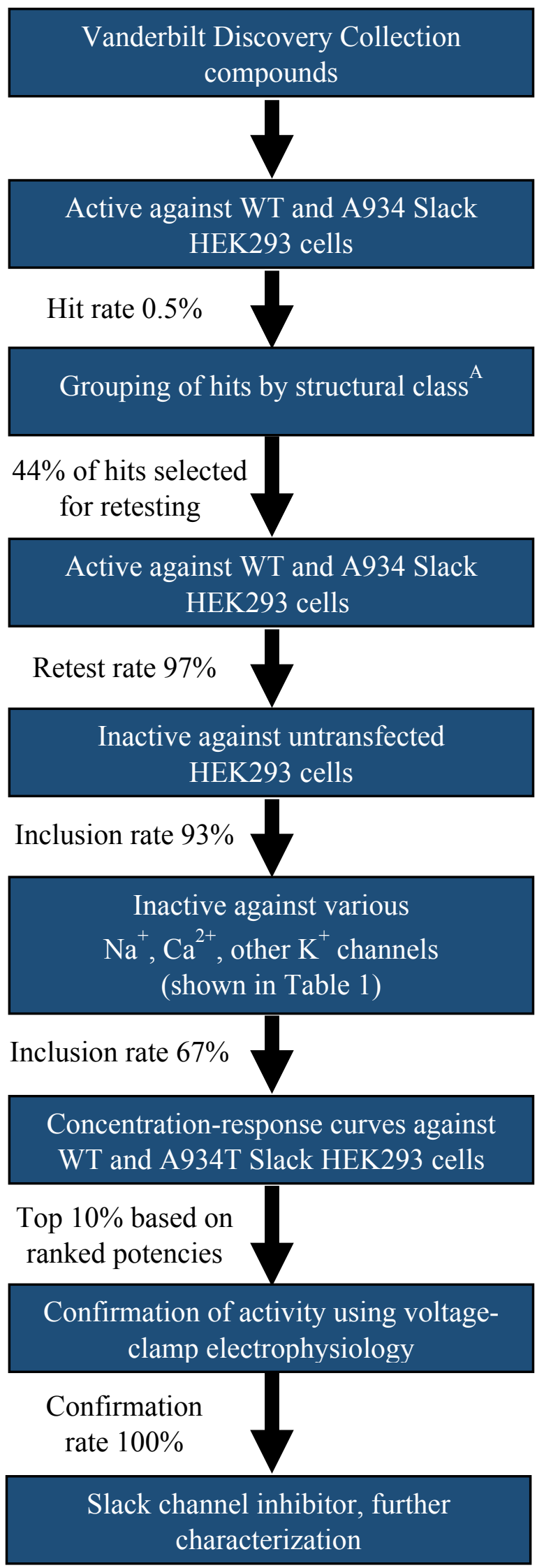

Figure S2: Diagram outlining the screening pipeline for Slack channels inhibitor discovery, with hit rate of initial screen and retesting rate listed.

${ }^{\mathrm{A}}$ Many hits fell into similar structural classes, in an effort to decrease the time and cost of retesting a subsection of hits were selected representatives of each structural class for retesting. 

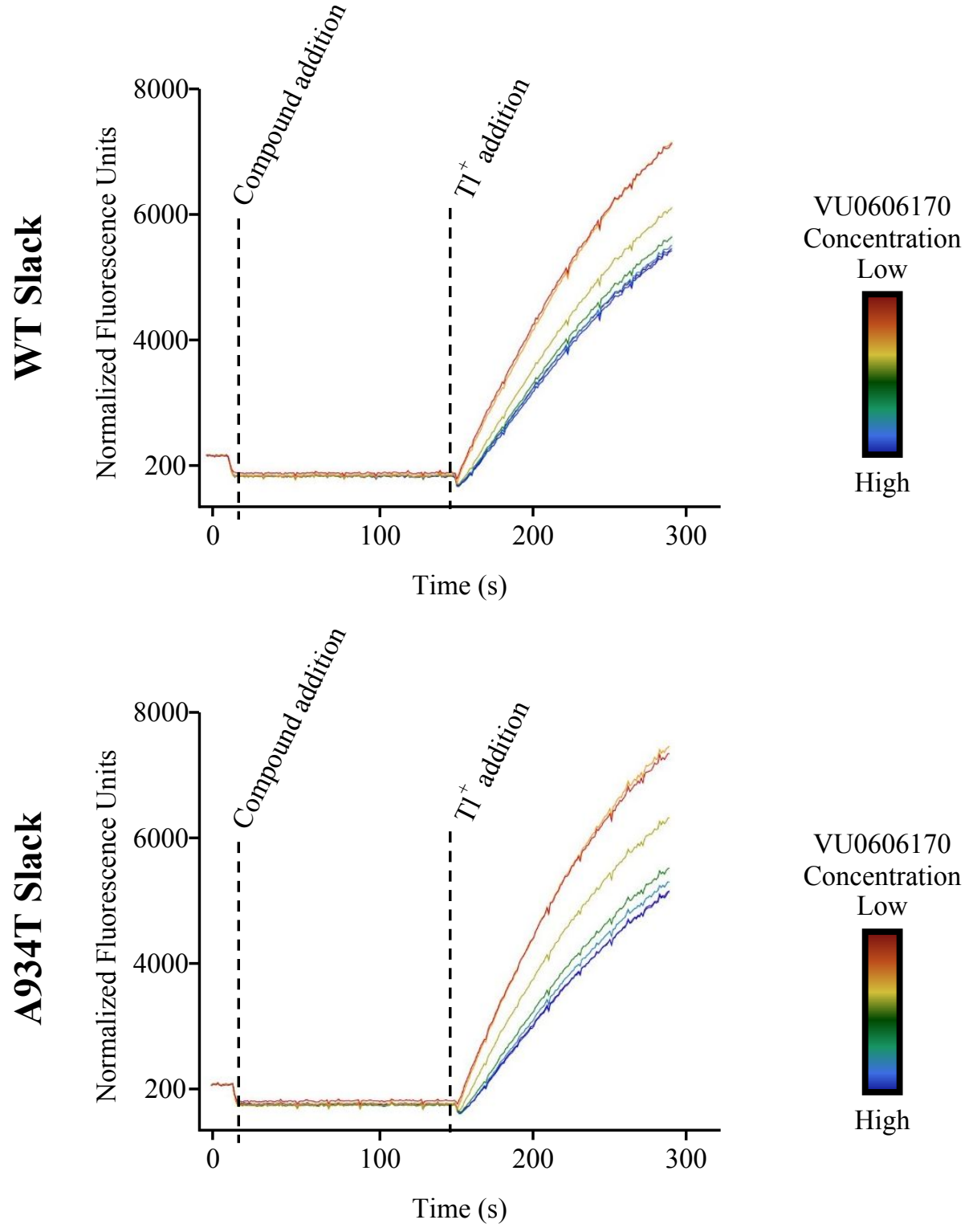

VU0606170

Concentration

Low

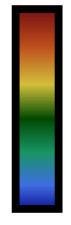

High

Figure S3: Concentration-dependent efficacy of VU0606170 measured using $\mathrm{Tl}^{+}$ flux. VU0606170 concentrations begin at $100 \mu \mathrm{M}$ and descend in 3-fold steps. Time of compound and $\mathrm{Tl}^{+}$addition represented as dotted lines. 


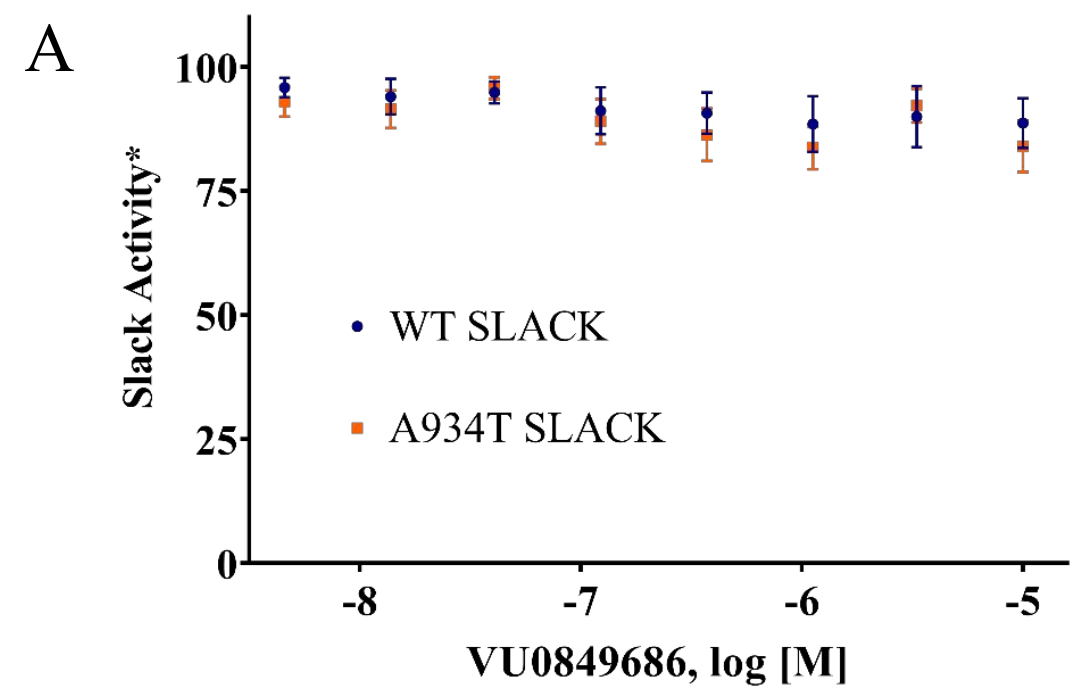

$\mathrm{B}$

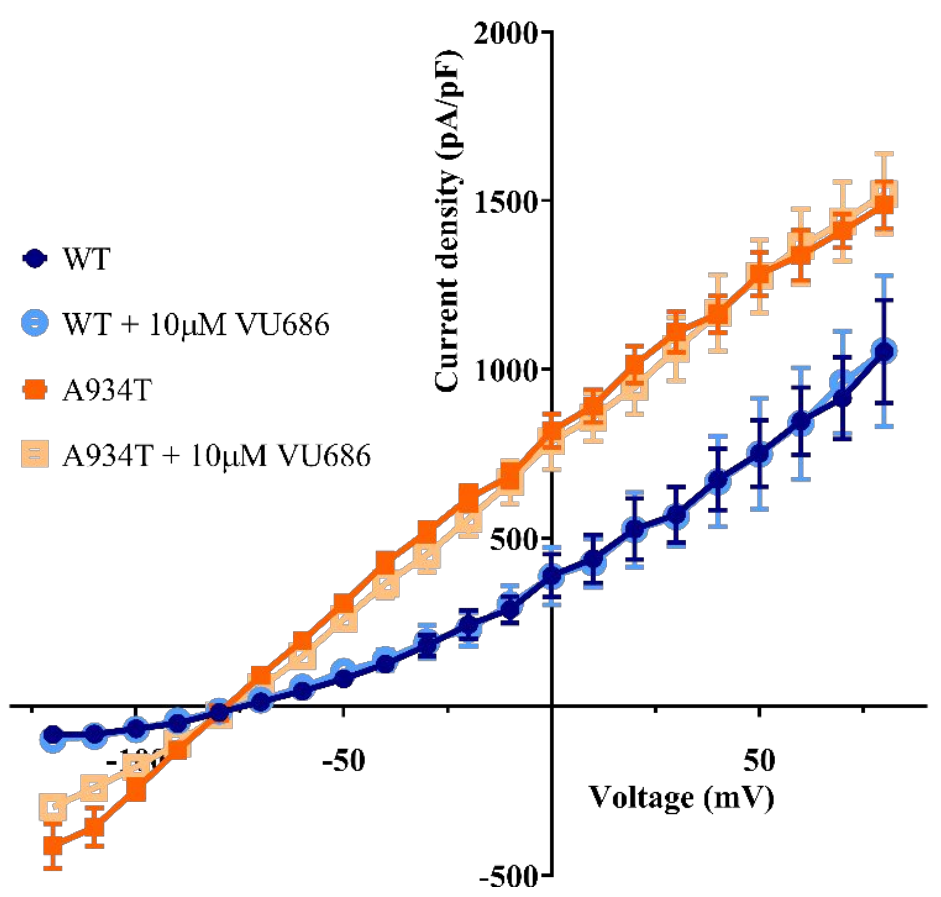

Figure S4: A) Concentration-response curves generated for WT Slack and A934T MMPSI-associated mutant Slack with VU0849686. CRCs arise from $\mathrm{Tl}^{+}$flux data obtained from monoclonal cell lines stably expressing WT Slack and A934T mutant Slack in ten separate trials. *VU0849686 data is normalized from $\mathrm{E}_{\max } \operatorname{VU170}(30 \mu \mathrm{M}) . \mathrm{EC}_{50}$ values could not be calculated, $\mathrm{n}=6$. B) Whole- cell voltage dependent outward Slack current evoked by stepping from $-120 \mathrm{mV}$ to $+80 \mathrm{mV}$ in $10-\mathrm{mV}$ increments in HEK-293 cells expressing WT or A934T Slack. The IV relationship for WT and A934T Slack were not affected by the addition of $30 \mu \mathrm{M}$ VU0849686. $(n=8-29)$ 

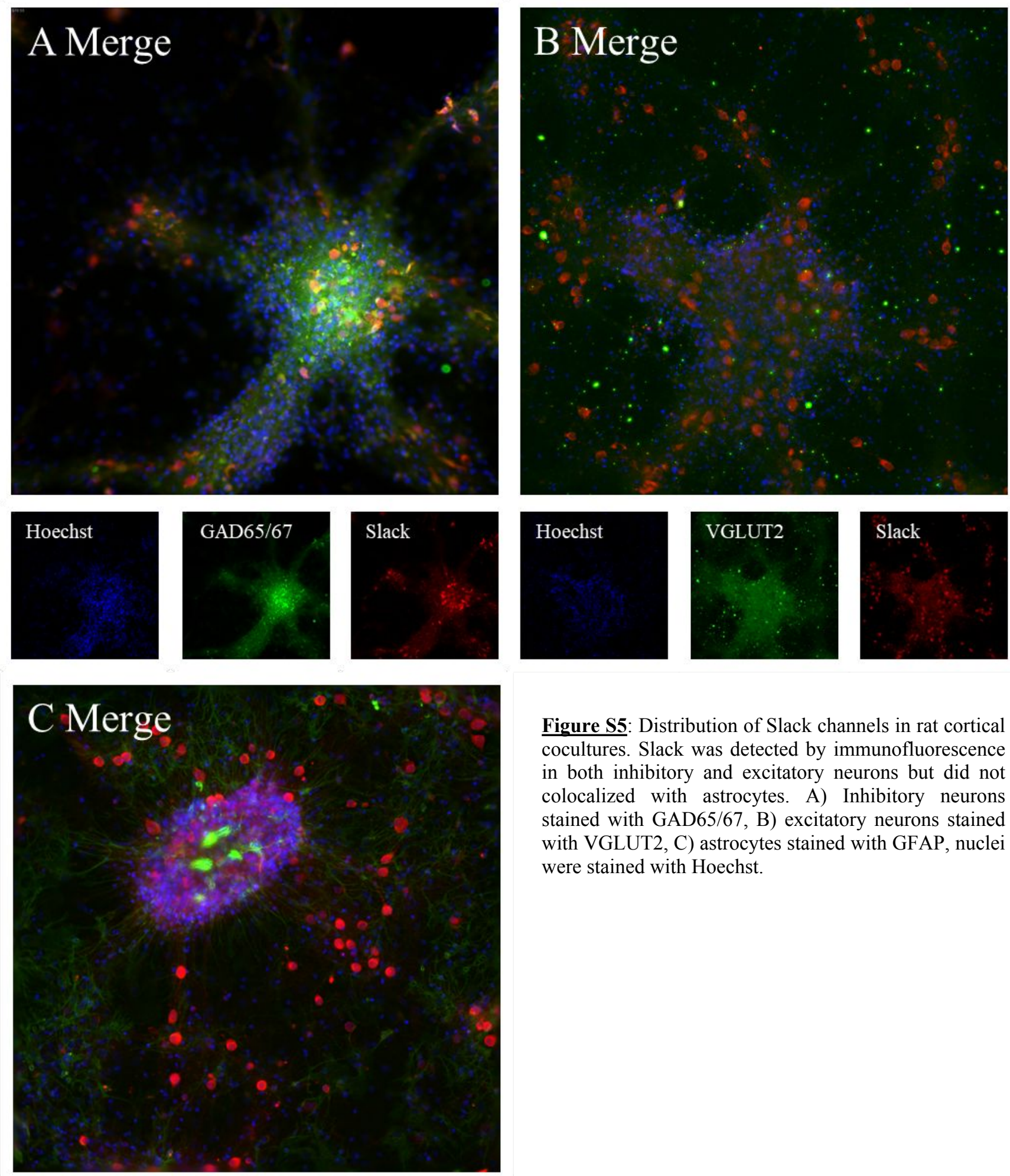

Figure S5: Distribution of Slack channels in rat cortical cocultures. Slack was detected by immunofluorescence in both inhibitory and excitatory neurons but did not colocalized with astrocytes. A) Inhibitory neurons stained with GAD65/67, B) excitatory neurons stained with VGLUT2, C) astrocytes stained with GFAP, nuclei were stained with Hoechst.

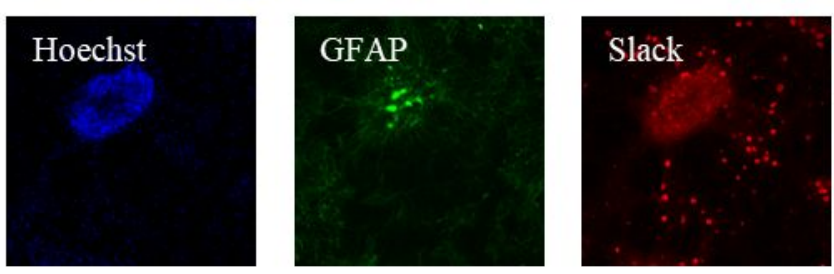

